(2) Open Access Full Text Article

REVIEW

\title{
Impact of Anti-Type 2 Inflammation Biologic Therapy on COVID-19 Clinical Course and Outcome
}

\author{
Dimitri Poddighe $\mathbb{D}^{1,2}$ \\ Elena Kovzel ${ }^{2}$
}

'Department of Medicine, Nazarbayev University School of Medicine, NurSultan, 010000 , Kazakhstan; ${ }^{2}$ Clinical Academic Department of Pediatrics, University Medical Center (UMC), NurSultan, 010000, Kazakhstan
Correspondence: Dimitri Poddighe Email dimitri.poddighe@nu.edu.kz

\begin{abstract}
SARS-CoV-2 pandemic had a general and deep impact on the clinical management of chronic diseases, including respiratory and allergic disorders. At the beginning of the pandemic, one of the main concerns was the potential impact of immunosuppressive/immunomodulatory drugs on COVID-19 clinical course. In this review, we aim to summarize and analyze the available clinical evidence from patients treated with anti-type 2 inflammation biologics (including anti-IgE, anti-IL-5 and anti-IL-4 agents), who developed COVID-19. Overall, the treatment with anti-Th2 biologics can be considered safe during COVID-19. It does not worsen the clinical course and outcome of COVID-19, and it may be actually protective somehow from developing severe forms. Moreover, patients treated with these biological agents do not seem to be more prone to get infected by SARS-CoV-2.
\end{abstract}

Keywords: COVID-19, SARS-CoV-2, omalizumab, dupilumab, benralizumab, mepolizumab, reslizumab, asthma, chronic spontaneous urticaria, biologics

\section{Introduction}

SARS-CoV-2 pandemic had a general and deep impact on the clinical management of most chronic diseases in adults and children. ${ }^{1,2}$ Especially in the first phases of this pandemic, a great concern was the potential effect of concomitant immunosuppressive/immunomodulatory therapies on the individual susceptibility, clinical course, and outcome of COVID-19. ${ }^{3}$

Rheumatic disorders immediately received a great attention to understand if the biological therapies could worsen the clinical course of COVID-19. In general, most international societies and experts recommended to continue the ongoing treatments in patients affected with rheumatic diseases under the appropriate medical supervision. ${ }^{4,5}$ A similar message was given for chronic and severe respiratory and allergic diseases needing a biological therapy. ${ }^{6-9}$ In the middle of the first wave of the pandemic (May 2020), a panel of international expert advised

to continue the administration of biological therapies during the COVID-19 pandemic in patients for whom such therapies are clearly indicated and have been effective

since no risk of increased (viral) infections susceptibility emerged from the previous clinical studies and considering the higher risk of asthma attacks (and increased need of steroidal drugs). ${ }^{10}$ 
Through this narrative review, after 1.5 years since the beginning of COVID-19 pandemic, we aim to summarize and analyze the available clinical evidence regarding the impact of the anti-type 2 inflammation biologics (including anti-IgE, anti-IL-5 and anti-IL-4 agents) on COVID-19 susceptibility and clinical course. Here, we reported all the consecutive clinical reports and series (according to the date of publication online) accumulated in this field, and we described the main findings, in order to make some preliminary observations and initial considerations.

\section{Omalizumab}

Omalizumab is an anti-IgE humanized monoclonal antibody, approved by the US Food and Drug Administration (FDA) and European Medicinal Agency (EMA) for patients with severe and difficult allergic asthma (starting from the age of 12 and 6 years, respectively), and for the therapy of chronic spontaneous urticaria (CSU) in patients whose symptoms cannot be appropriately controlled through the conventional drugs. ${ }^{11}$

The first description of an asthmatic patient on omalizumab diagnosed with SARS-CoV-2 infection was published by Lommatzsch et al (online publication: June 2020): this 52-year-old man did not develop any pulmonary complications and did not show any significant differences in asthma control or biomarkers, as compared to his previous clinical course before the pandemic. ${ }^{12}$

In the same period (online publication: August 2020), Criado et al reported the likely positive impact of omalizumab on COVID-19 clinical course of a patient who received it during this infection for the first time. Indeed, because of the aggravation of refractory CSU (despite a combined therapy including steroids), this 54-year-old female patient diagnosed with COVID-19 pneumonia, was treated with omalizumab: a prompt and complete resolution of urticarial manifestations was achieved, which was also accompanied with a concomitant improvement of COVID-19 clinical course. $^{13}$

Several authors suggested that omalizumab may confer some protection against severe forms of COVID-19 or even help to manage them. ${ }^{14-16}$ For instance, Baldallo et al (online publication: July 2020) described a mild form of COVID-19 despite a concomitant chronic renal disease requiring hemodialysis, in a patient affected with MPO-ANCA positive urticarial vasculitis and, thus, receiving omalizumab for this off-label medical indication. ${ }^{17}$
After these first case reports, a small case series provided evidence in the same direction, namely that omalizumab does not seem to have a negative impact on COVID-19. Ayhan et al (online publication: December 2020) described three CSU patients on omalizumab infected with SARS-CoV-2, who fully recovered without any disruption of the biological therapeutic regimen, even though 2 of them experienced lung involvement during the infection. ${ }^{18}$

Conversely, the analysis of COVID-19 patients included in the Dutch Severe Asthma Registry RAPSODI (Registry of Adult Patients with Severe asthma for Optimal Disease management) published by Eger et al (online publication: December 2020) actually raised some concerns. These authors collected all cases of COVID-19 between March 1, 2020, and April 30, 2020, among patients who were treated with biologics. Nine of these 634 severe asthma patients on biological treatment $(1.42 \%)$ were diagnosed with COVID-19, and seven required hospitalizations. Among these, only two patients received omalizumab and both were hospitalized and required intensive care, but they recovered. However, among all those patients hospitalized for COVID-19, they were those showing the highest number of known risk factors for developing severe forms: both patients were affected with obesity, diabetes mellitus and cardiovascular disease. ${ }^{19}$

Larger clinical studies investigating omalizumab in COVID-19 patients were published only recently. Passante et al (online publication: August 2021) described a mild clinical course of SARS-CoV-2 infection in seven adult CSU patients treated with omalizumab: no one had to stop the biological treatment and three of them were even completely asymptomatic. ${ }^{20}$

Shortly after, Muntean et al (online publication: September 2021) analyzed their case series of 218 adult patients affected with CSU: 149 patients had moderate-tosevere CSU, and 25 of them were treated with omalizumab. Among the 72 patients infected with SARS-CoV-2, $10(13,9 \%)$ were on treatment with omalizumab and all developed moderate-severe COVID-19. However, by reanalyzing the data provided by the authors, this $13.9 \%$ percentage of moderate-severe COVID-19 in patients treated with omalizumab (who concomitantly also received fourfold dose increase of $\mathrm{H} 1$-antihistamines) was even lower than patients receiving only $\mathrm{H} 1$-antihistamines $(\mathrm{n}=26,36 \%)$ at the standard dosage. Therefore, in this study, there is no evidence of increased COVID-19 
severity in patients treated with omalizumab compared to patients receiving the conventional treatment only. Notably, the percentage of moderate-severe COVID-19 was $9.8 \%(n=5)$ in completely untreated CSU patients. ${ }^{21}$

As regards omalizumab and asthma, Aksu et al (online publication: September 2021) reviewed their case series of adult severe patients using biological agents, which also included 62 patients treated with omalizumab. Among these, 9 patients were diagnosed with COVID-19 and, notably, no one showed a severe course. ${ }^{22}$

In the same month, Abduelmula et al (online publication: September 2021) reported that among 184 patients (presumably all adults) on omalizumab for CSU or asthma, followed in several Canadian centers and included in the Patient Support Program, no COVID-19 cases were identified in the study period (February 1, 2020 - April 30, 2021). ${ }^{23}$

Finally, Bostan et al (online publication: October 2021) described 233 adult patients $(44.76 \pm 14.16$ years, range: 18-80) affected with CSU, which were analyzed according to the type of treatment as well. In detail, they did not find any statistically significant difference between patients treated with omalizumab in addition to oral antihistamines and those receiving only oral antihistamines, in terms of SARS-CoV-2 RT-PCR positivity. ${ }^{24}$

\section{Anti-IL-5 Biologics}

This class of biologics includes three main molecules that are mainly indicated to treat severe eosinophilic asthma and chronic respiratory diseases: mepolizumab, reslizumab and benralizumab. Mepolizumab and Reslizumab are humanized monoclonal antibodies targeting human interleukin-5 (IL-5): they both block the binding of IL-5 to the IL-5 receptor complex, which is mainly expressed on the eosinophil cell surface. Benralizumab antagonizes the IL-5 signaling by targeting the IL-5 alpha receptor: it is a humanized afucosylated monoclonal antibody that, through the binding to the IL-5 receptor on the cell surface, induces eosinophil apoptosis by antibody-dependent cell-mediated cytotoxicity (ADCC). As a final result, all these biologics lead to eosinophil depletion. ${ }^{10}$

Renner et al (online publication: June 2020) reported the first case of a patient on treatment with an anti-IL-5 agent, who experienced a mild COVID-19 course. He was a 41-year-old asthmatic male treated with benralizumab. ${ }^{25}$ Later, these authors (online publication: October 2020) also described a second patient affected with asthma and chronic rhinosinusitis with nasal polyps (CRSwNP), who was infected with SARS-CoV-2 during the treatment with benralizumab. Despite the older age (66 years), he did not develop any pulmonary complications, and did not require any increase in asthma medications. ${ }^{26}$

Heffler et al (online publication: August 2020) published the data collected from Severe Asthma Network in Italy (SANI) during the first wave of pandemic. Among the population of severe asthmatics $(n=1504), 65 \%$ $(n=978)$ of them were treated with biologics (anti-IL5 agents: $52.9 \%, \mathrm{n}=517$ after extrapolation; omalizumab: $47.1 \%$, thus $n=461$, after extrapolation). In total, the authors reported 26 patients diagnosed with COVID-19 at the time of the analysis, of whom 21 were on biological treatment. Based on this information provided by the authors, the COVID-19 diagnostic rate was $2.15 \%$ and $0.95 \%$ in patients with and without biological therapy, respectively; however, this calculation (and the relative statistical analysis) was not clearly expressed by the authors. In detail, the authors reported the following biological therapies in these 21 COVID-19 patients: omalizumab $(n=6)$, mepolizumab $(n=14)$, and benralizumab $(n=1)$; which corresponds to a percentage of COVID-19 cases of $1.3 \%$ and $2.9 \%$ for omalizumab and IL-5 antagonists, respectively, as emphasized by the authors themselves. ${ }^{27}$ Apparently, one might observe that patients on omalizumab seems to be less affected with SARS-CoV-2 infection, but no statistical analysis was available in this article. The percentage of COVID-19 cases was $1.7 \%$ for the whole cohort of asthmatic patients and $2.15 \%$ for the patients treated with biologics, overall. However, this difference may be related to the biological treatment itself or to a greater attention for these patients in terms of molecular testing, compared to those patients treated with conventional therapy. Finally, among the 26 COVID-19 patients, two fatal outcomes were observed, one in the group of patients on biological therapy (and, in detail, mepolizu$\mathrm{mab}$ ) and one in the group of patients treated without biologics. In this regard, no definitive conclusion can be made at all, of course. However, as emphasized by the authors, the COVID-19 fatality rate in this cohort patients on biological therapy resulted to be $7.7 \%$, which was lower than that in the general population $(14.5 \%$ in Italy at the time of the study), which may suggest that the mortality should not be increased by these biologics. ${ }^{27}$

COVID-19 patients on treatment with anti-IL-5 agents were also described in the study by Eger et al (online publication: December 2020), which was analyzed in the previous section. Actually, among those nine severe 
asthma patients treated with biologics and diagnosed with COVID-19 (which were $1.42 \%$ of the entire cohort), six patients received an anti-IL-5 biologic (mepolizumab, $\mathrm{n}=3$; reslizumab, $\mathrm{n}=1$; benralizumab, $\mathrm{n}=2$ ). All of them except one needed to be hospitalized for oxygen therapy and one (mepolizumab) unfortunately died. ${ }^{19}$

After these relatively larger experiences, some additional case reports were published. Kroes et al (online publication: April 2021) described another asthmatic patient (64-year-old woman) on treatment with benralizumab and SARS-CoV-2 infected. This patient experienced a rapid deterioration of the respiratory function after the COVID-19 diagnosis and was admitted to the intensive care unit (ICU). The treatment with benralizumab was maintained during the ICU stay; however, this patient had a severe clinical course anyway, since tracheotomy was needed at day-31 from ICU admission, in order to facilitate the weaning off the mechanical ventilation. The therapy with benralizumab resulted to be well tolerated by this patient who was on mechanical ventilation, but it does not seem to have significantly and/or positively impacted on the COVID-19 clinical course. $^{28}$

To conclude, some additional and interesting experiences with benralizumab in the clinical context of drug rash with eosinophilia and systemic symptoms (DRESS) deserve to be mentioned. Schmid-Grendelmeier et al (online publication: October 2020) reported the use of benralizumab in 2 non-asthmatic patients (a 54-year-old woman and a 58-year-old man) who developed DRESS during severe COVID-19, which required the admission to the ICU for acute respiratory distress syndrome. Because of worsening eosinophilia and deterioration of organs function, the treatment with benralizumab was implemented. ${ }^{29}$ Eosinophilia can develop during COVID19 clinical course and, notably, it may be associated to some biological therapies, such as IL-6 antagonists; ${ }^{30}$ however, the use of tocilizumab was not reported in these clinical cases. Both patients showed improvement of DRESS manifestations in the 2 weeks following this biological treatment; however, only one patient was reported to be alive 28 days after the administration of benralizumab, whereas the other patient died because of disseminated intravascular coagulation secondary to COVID-19 at day-17 from the biological treatment. No specific and precise information is provided about the course of COVID-19 in each patient; however, as said, one died because of this infection, despite the therapy with benralizumab. ${ }^{29}$

Another similar case of COVID-19-related DRESS was reported by Mesli et al (online publication: July 2021). This complication occurred 38 days after admission to the ICU for severe acute respiratory syndrome. Despite the conventional treatment for DRESS, the patient developed hemophagocytic lymphohistiocytosis and the decision to start benralizumab was made. A rapid decrease of eosinophilia within 2 days and the resolution of hemophagocytic lymphohistiocytosis was observed, in addition to the rapid improvement of both organ dysfunction and skin lesions. The final outcome was positive: he withdrew dialysis and was discharged from the ICU 4 weeks after benralizumab treatment. ${ }^{31}$

\section{Dupilumab}

Dupilumab is a recombinant monoclonal antibody directed against the alpha-chain of the IL-4 receptor (IL-4Ra), which is shared with IL-13 receptor as well; therefore, this drug is able to inhibit the signaling of both these interleukins. This therapy is associated with reduced levels of several Th2 chemokines, total IgE and allergen-specific $\operatorname{IgE}^{32}$

A mild clinical course of COVID-19 in a 53-year-old patient treated with dupilumab (because of CRSwNP) was first reported by Forster-Ruhrmann et al (online publication: May 2020) at the beginning of the pandemic. ${ }^{33}$

Ferrucci et al (online publication: June 2020) reported two adult patients (40-year-old man and 56-year-old woman) affected with severe atopic dermatitis (AD) since childhood and, therefore, treated with dupilumab. These patients continued this therapy even when they were diagnosed with COVID-19, whose clinical course was mild. Both patients had a close contact who was diagnosed with COVID-19 as well: notably, these contacts both died because of the complications of interstitial pneumonia, unlike these patients treated with dupilumab. ${ }^{34}$

Eventually, Caroppo et al (online publication: June 2020) reported an elderly patient (72-year-old man) on treatment with dupilumab for severe $\mathrm{AD}$, who was detected as SARSCoV-2 positive during a screening campaign. Despite a prolonged positivity of the nasopharyngeal swab for SARS-CoV-2 for around 9 weeks, this patient did not develop COVID-19-related manifestations throughout all this period, in addition to being free of AD manifestations. ${ }^{35}$ Interestingly, a long persistence ( 9 weeks after the onset of symptoms) of the virus in the naso-pharyngeal swab, was also described by 
Bhalla et al (online publication: August 2020) in a 23-year-old female patient treated with dupilumab for asthma. ${ }^{36}$

Rossi et al (online publication: June 2020) also reported 2 patients diagnosed in March 2020 with COVID-19 based on a telephone survey including 71 adult patients treated with dupilumab for $\mathrm{AD}$. Both patients recovered without any sequelae, even if one required non-invasive ventilation during the acute phase; however, this latter patient was a 53-year-old woman with multiple comorbidities, including asthma, hypertension, severe obesity, and depressive syndrome. ${ }^{37}$

As reported by Ordonez et al (online publication: September 2020), the first case of COVID-19 in a patient treated with dupilumab in Latin America dates back to May 2020. A 22-year-old male patient was diagnosed with asymptomatic SARS-CoV-2 infection. ${ }^{38}$ Later, the same authors described another COVID-19 case in a young adult (27 years old) patient on dupilumab, who was also HIV-positive. Like in the previous publication, these authors did not provide a detailed clinical description, but his clinical course was reported to be mild and without need of hospitalization. ${ }^{39}$

Similarly, Tanabe et al (online publication: November 2020) first reported a 57-year-old asthmatic man on dupilumab from Japan, who was diagnosed with SARS-CoV-2 infection because of a close contact. Even if at the diagnosis he was asymptomatic, later he developed pneumonia from which he recovered at the end; notably, like some of the previous reports, the virus was detected for at least 4 weeks from the diagnosis. ${ }^{40}$

As regards larger studies, one COVID-19 patient on dupilumab was also present in the study by Eger et al (online publication: December 2020), which was described in the previous sections. This asthmatic patient did not require hospitalization at all. ${ }^{19}$

However, Chiricozzi et al (online publication: March 2021) reported the first cases series of COVID-19 patients receiving dupilumab. Among the 1831 adult patients affected with moderate-severe $\mathrm{AD}$, who were included in the Italian multicentric DA-COVID registry, $85 \%$ were treated with dupilumab, which was prescribed as monotherapy in most cases. In the whole cohort, 16 COVID-19 cases were observed and 15 were receiving dupilumab. During the infection, eight continued dupilumab and seven discontinued it. No COVID-19 related complications were observed in patients who continued dupilumab, whereas there is no information about the clinical course in the other seven patients. In general, no detailed description of the dynamics of the COVID-19 clinical episode is present in this article, unfortunately. ${ }^{41}$

Dupilumab can be also used in adolescents with moderate-severe AD. Stingeni et al (online publication: June 2021) described a 16-week real-life experience with dupilumab started from January to October 2020 in 19 adolescents (range 13-17 years). No greater susceptibility to SARS-CoV-2 infection was noticed: indeed, only one case was detected, whose clinical course was without any

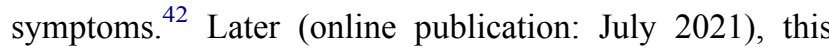
same Italian group described 9 adolescents undergoing 32-week dupilumab treatment during the pandemic. Again, only one case of COVID-19 at week-10 was mentioned, and this is supposed to be the same reported in the previous article. ${ }^{43}$

Finally, a mild course has been recently observed in three COVID-19 cases from Poland reported by Ceryn et al (online publication: August 2021) among their young adults affected with $\mathrm{AD}$ and treated with dupilumab. All these three patients were young (27, 19 and 17 years old). ${ }^{44}$

\section{Impact of Anti-Th2 Therapy on COVID-19: Potential Mechanisms}

Based on the previous discussion, the anti-Th2 inflammation therapies may be able to provide some beneficial effects in treated patients developing COVID-19, as schematically summarized in Table 1. Further research and, in detail, controlled clinical studies are required to confirm this initial evidence, which is basically supported by case reports and series only. However, we may briefly discuss some potential and general mechanisms by which these biologics could positively affect the clinical response to SARS-CoV-2 infection in patients receiving them for allergic and/or respiratory disorders.

First of all, the primary defense against respiratory viruses relies on the activation of the innate immunity through the production of type I interferons, which can limit the viral spread. Notably, plasmacytoid dendritic cells (pDCs) are the primary blood source of IFN- $\alpha$ / $\beta .^{45,46} \mathrm{~A}$ reduction in the production of these cytokines by pDCs has been observed in atopic patients, and this impairment resulted to correlate with serum $\mathrm{IgE}$ levels in one study. This finding suggested that pDCs antiviral responses may be suppressed in atopic patients, and this may represent one of the mechanisms by which atopy is supposed to increase the susceptibility to respiratory viral 
Table I Case Reports or Original Articles Providing Information on COVID-19 Outcome in Patients Treated with Anti-Th2 Biologics

\begin{tabular}{|l|c|c|c|c|}
\hline Biologics & Articles (N) & COVID-19 Patients (N) & Moderate-Severe COVID-19 (N) & Fatal Outcome (N) \\
\hline Omalizumab & 8 & 34 & 10 & 0 \\
\hline Mepolizumab & 2 & 17 & 6 & 2 \\
\hline Benralizumab & $5^{*}$ & 6 & 1 & 0 \\
\hline Reslizumab & 1 & 1 & 2 & 0 \\
\hline Dupilumab & 11 & 21 & 3 & 0 \\
\hline
\end{tabular}

Notes: *The cases (and related articles) where benralizumab was started to treat DRESS have not been included, even though these experiences were reported in the discussion.

infections. ${ }^{47}$ Therefore, omalizumab treatment could improve type I IFNs responses to respiratory viruses, which may also occur during COVID-19. This effect has been supported by a pediatric study describing improved IFNs responses after a short-term treatment with omalizumab in patients affected with severe asthma. ${ }^{48}$

In general, anti-Th2 inflammation therapies may favor viral infections control by reducing this type of immunological polarization and, thus, indirectly promoting the switch toward other T-helper populations and, in detail, Th1 lymphocytes, which can better fight against viruses. Indeed, the classical Th1 response could be defective during acute phase of COVID-19. ${ }^{49}$ Severe COVID-19 is characterized by systemic and uncontrolled inflammation, which follows an initial increment of all cytokines, and seems to be associated to a decrease of Th1 response, overall. Moreover, severe COVID-19 patients also showed a marked Th2 immune response in the context of the associated cytokine storm: an increase of IL-5, IL-13, eotaxin-2, IgE, and eosinophils was observed in these patients, which also correlated with the severity of the clinical course. $^{50,51}$ Even IL-4 (which is the main driver of the Th2 lymphocyte polarization) $)^{52,53}$ was reported to be elevated in severe COVID-19 patients admitted to the intensive care unit. ${ }^{54}$ Therefore, it is plausible that the interference with IL-5 and IL-4(/IL-13) signaling given by mepolizumab/reslizumab/benralizumab and dupilumab, respectively, may somehow and indirectly favor Th1 responses and, thus, the viral clearance by the adaptive immune system.

As mentioned above, one of the main aspects of COVID-19 severe forms is a systemic and uncontrolled inflammation leading to multi-organ dysfunction. $\operatorname{IgE}$ might also play some immunomodulatory role in this context. During respiratory viral infections, the production of specific IgE against different viruses has been reported, and IgE may contribute in the immune response to these infections at later stages, maybe by activating mast cells and basophils and/or by modulating the production of proinflammatory cytokines, such as TNF- $\alpha$ and IL- $6 .^{53,55,56}$ Anti-IgE therapy may be effective in this regard, considering the effect on mast cells and basophils production and activation. ${ }^{57,58}$ Actually, eosinophils may also have a direct role in COVID-19-related inflammation. ${ }^{59}$ Indeed, during the first phases of respiratory infections, an active migration of circulating eosinophils from the peripheral blood to target tissues can occur, and eosinophils are known to perform some antiviral functions. ${ }^{60,61}$ However, eosinophils could complicate COVID-19 immunopathology at a later stage. In a recent post-mortem series of COVID-19 patients, eosinophils were found in the alveolar septs, ${ }^{62}$ and several case reports of COVID-19 patients undergoing bronchoalveolar lavage and/or lung biopsy showed significant eosinophilic infiltrates in lungs. ${ }^{63,64}$ Taken together, all these observations may provide an additional hypothesis explaining why the use of both omalizumab and anti-Th2 cytokines therapies could further improve the clinical course of COVID-19.

As a very final remark, considering the mass vaccination campaign for COVID-19 all over the world, it may be worth just mentioning two short clinical reports describing patients who received clinical benefit from omalizumab (two patients) and mepolizumab (one patient) to prevent anaphylactoid reactions and control of severe asthma exacerbations following mRNA COVID-19 vaccine, respectively. ${ }^{65,66}$

\section{Conclusion}

Overall, the treatment with anti-Th2 biologics can be considered safe during COVID-19. It does not worsen 
the clinical outcome of COVID-19 and it may be even protective somehow from severe forms. Moreover, patients treated with these biological agents are not likely to be more prone to be infected by SARS-CoV2. According to our case-based review, the only two COVID-19-related deaths were observed in patients treated with mepolizumab, as showed in Table 1. Indeed, the proportion of patients with moderate to severe forms of COVID-19 resulted to be greater in patients treated with anti-IL-5 agents, compared to the other classes. Notably, dupilumab was characterized by a very low rate of clinically significant forms of COVID-19. As regards omalizumab, the concomitant treatment with antihistamine drugs (especially if higher than the standard dose) should be kept in consideration for the correct interpretation of the related numbers. Of course, controlled studies are needed to make final conclusions on the safety and even benefit that these biological agents may provide to the clinical course of COVID-19 in specific clinical settings.

\section{Abbreviations}

IgE, immunoglobulin E; IL-4, interleukin-4; IL-5, interleukin-5; IL-13, interleukin-13; ICU, intensive care unit; CSU, chronic spontaneous urticaria; PCR, polymerase chain reaction; $\mathrm{AD}$, atopic dermatitis; DRESS, drug rash with eosinophilia and systemic symptoms; CRSwNP, chronic rhinosinusitis with nasal polyps.

\section{Acknowledgment}

The APC was funded by the Social Policy Grant of Nazarbayev University.

\section{Disclosure}

The authors report no conflicts of interest in this work.

\section{References}

1. Tiotiu A, Chong Neto H, Bikov A, et al. Impact of the COVID-19 pandemic on the management of chronic noninfectious respiratory diseases. Expert Rev Respir Med. 2021;15(8):1035-1048. doi:10.1080/17476348.2021.1951707

2. Sabetkish N, Rahmani A. The overall impact of COVID-19 on healthcare during the pandemic: a multidisciplinary point of view. Health $\mathrm{Sci}$ Rep. 2021;4:e386. doi:10.1002/hsr2.386

3. Robinson PC, Yazdany J. The COVID-19 global rheumatology alliance: collecting data in a pandemic. Nat Rev Rheumatol. 2020;16 (6):293-294. doi:10.1038/s41584-020-0418-0

4. Misra DP, Agarwal V, Gasparyan AY, Zimba O. Rheumatologists' perspective on coronavirus disease 19 (COVID-19) and potential therapeutic targets. Clin Rheumatol. 2020;39(7):2055-2062. doi:10.1007/ s10067-020-05073-9
5. Ceribelli A, Motta F, De Santis M, et al. Recommendations for coronavirus infection in rheumatic diseases treated with biologic therapy. $J$ Autoimmun. 2020;109:102442. doi:10.1016/j. jaut.2020.102442

6. Wollenberg A, Flohr C, Simon D, et al. European task force on Atopic Dermatitis statement on severe acute respiratory syndrome coronavirus 2 (SARS-Cov-2) infection and atopic dermatitis. $J$ Eur Acad Dermatol Venereol. 2020;34(6):e241-e242. doi:10.1111/ jdv. 16411

7. Vultaggio A, Agache I, Akdis CA, et al. Considerations on biologicals for patients with allergic disease in times of the COVID-19 pandemic: an EAACI statement. Allergy. 2020;75(11):2764-2774. doi:10.1111/all.14407

8. Klimek L, Pfaar O, Worm M, et al. Use of biologicals in allergic and type-2 inflammatory diseases during the current COVID-19 pandemic: position paper of Ärzteverband Deutscher Allergologen $(\mathrm{AeDA})^{\mathrm{A}}$, Deutsche Gesellschaft für Allergologie und Klinische Immunologie (DGAKI) ${ }^{\mathrm{B}}$, Gesellschaft für Pädiatrische Allergologie und Umweltmedizin $(\mathrm{GPA})^{\mathrm{C}}$, Österreichische Gesellschaft für Allergologie und Immunologie (ÖGAI) $)^{\mathrm{D}}$, Luxemburgische Gesellschaft für Allergologie und Immunologie $(\mathrm{LGAI})^{\mathrm{E}}$, Österreichische Gesellschaft für Pneumologie (ÖGP) ${ }^{\mathrm{F}}$ in co-operation with the German, Austrian, and Swiss ARIA groups ${ }^{\mathrm{G}}$, and the European Academy of Allergy and Clinical Immunology $(\text { EAACI })^{\mathrm{H}}$. Allergol Select. 2020;4:53-68. doi:10.5414/ALX02166E

9. Ricardo JW, Lipner SR. Considerations for safety in the use of systemic medications for psoriasis and atopic dermatitis during the COVID-19 pandemic. Dermatol Ther. 2020;33(5):e13687. doi:10.1111/dth. 13687

10. Morais-Almeida M, Aguiar R, Martin B, et al. COVID-19, asthma, and biological therapies: what we need to know. World Allergy Organ J. 2020;13(5):100126. doi:10.1016/j.waojou.2020.100126

11. Poddighe D, Brambilla I, Licari A, Marseglia GL. Omalizumab in the therapy of pediatric asthma. Recent Pat Inflamm Allergy Drug Discov. 2018;12(2):103-109. doi:10.2174/1872213X12666180430161351

12. Lommatzsch M, Stoll P, Virchow JC. COVID-19 in a patient with severe asthma treated with Omalizumab. Allergy. 2020;75(10)::27052708. doi:10.1111/all.14456

13. Criado PR, Criado RFJ, Pincelli TP, Yoshimoto TA, Naufal GGA, Abdalla BMZ. Chronic spontaneous urticaria exacerbation in a patient with COVID-19: rapid and excellent response to omalizumab. Int J Dermatol. 2020;59(10):1294-1295. doi:10.1111/ijd.15134

14. Liu S, Zhi Y, Ying S. COVID-19 and asthma: reflection during the pandemic. Clin Rev Allergy Immunol. 2020;59(1):78-88. doi:10.1007/s12016-020-08797-3

15. Criado PR, Pagliari C, Criado RFJ, Marques GF, Belda W Jr. What the physicians should know about mast cells, dendritic cells, urticaria, and omalizumab during COVID-19 or asymptomatic infections due to SARS-CoV-2? Dermatol Ther. 2020;33:e14068. doi:10.1111/ dth. 14068

16. Abdelmaksoud A, Goldust M, Vestita M. Omalizumab and COVID-19 treatment: could it help? Dermatol Ther. 2020;33: e13792. doi:10.1111/dth.13792

17. Baldallo C, León Román JC, Serón D, et al. COVID-19 in a patient with hypocomplementemic urticarial syndrome and MPO-ANCA vasculitis on hemodialysis treated with omalizumab. Nefrologia. 2021;41(3):354-356. doi:10.1016/j.nefroe.2020.07.003

18. Ayhan E, Öztürk M, An İ, Bekçibaşi M. COVID-19 infection under omalizumab therapy for chronic spontaneous urticaria: three cases. Int J Dermatol. 2021;60(2):253-254. doi:10.1111/ijd.15379

19. Eger K, Hashimoto S, Braunstahl GJ, et al. Poor outcome of SARS-CoV2 infection in patients with severe asthma on biologic therapy. Respir Med. 2020;177:106287. doi:10.1016/j.rmed.2020.106287

20. Passante M, Napolitano M, Dastoli S, et al. Safety of omalizumab treatment in patients with chronic spontaneous urticaria and COVID-19. Dermatol Ther. 2021. doi:10.1111/dth.15111 
21. Muntean IA, Pintea I, Bocsan IC, Dobrican CT, Deleanu D. COVID19 disease leading to chronic spontaneous urticaria exacerbation: a Romanian retrospective study. Healthcare. 2021;9(9):1144. doi:10.3390/healthcare9091144

22. Aksu K, Demir Ş, Topel M, et al. COVID-19 in patients with severe asthma using biological agents. Tuberk Toraks. 2021;69(3):433-436. doi: $10.5578 /$ tt.20219721

23. Abduelmula A, Georgakopoulos JR, Mufti A, et al. Incidence of COVID-19 in patients with chronic idiopathic urticaria and asthma on Omalizumab: a multicentre retrospective cohort study. J Cutan Med Surg. 2021:120347542110497. doi:10.1177/12034754211049707

24. Bostan E, Zaid F, Karaduman A, et al. The effect of COVID-19 on patients with chronic spontaneous urticaria treated with omalizumab and antihistamines: a cross-sectional, comparative study. $J$ Cosmet Dermatol. 2021;20(11):3369-3375. doi:10.1111/jocd.14484

25. Renner A, Marth K, Patocka K, Pohl W. COVID-19 in a severe eosinophilic asthmatic receiving benralizumab - a case study. J Asthma. 2021;58(9):1270-1272. doi:10.1080/02770903.2020.1781165

26. Renner A, Marth K, Patocka K, Idzko M, Pohl W. COVID-19 in two severe asthmatics receiving benralizumab: busting the eosinophilia myth. ERJ Open Res. 2020;6(4):00457-2020. doi:10.1183/ 23120541.00457-2020

27. Heffler E, Detoraki A, Contoli M, et al.; SANI Working Group. COVID-19 in Severe Asthma Network in Italy (SANI) patients: clinical features, impact of comorbidities and treatments. Allergy. 2021;76(3):887-892. doi:10.1111/all.14532

28. Kroes JA, Zielhuis SW, Bethlehem C, Ten Brinke A, Van Roon EN. Administration of benralizumab in a patient with severe asthma admitted to the intensive care unit with COVID-19 pneumonia: case report. Eur J Hosp Pharm. 2021. doi:10.1136/ejhpharm-2020002660

29. Schmid-Grendelmeier P, Steiger P, Naegeli MC, et al. Benralizumab for severe DRESS in two COVID-19 patients. J Allergy Clin Immunol Pract. 2021;9(1):481-483. doi:10.1016/j.jaip.2020.09.039

30. Sernicola A, Carnicelli G, Di Fraia M, et al. 'Toxic erythema' and eosinophilia associated with tocilizumab therapy in a COVID-19 patient. J Eur Acad Dermatol Venereol. 2020;34(8):e368-e370. doi:10.1111/jdv.16620

31. Mesli F, Dumont M, Soria A, et al. Benralizumab: a potential tailored treatment for life-threatening DRESS in the COVID-19 era. J Allergy Clin Immunol Pract. 2021;9:3529-3531.

32. Ricciardolo FLM, Bertolini F, Carriero V. The role of Dupilumab in severe asthma. Biomedicines. 2021;9(9):1096. doi:10.3390/ biomedicines 9091096

33. Förster-Ruhrmann U, Szczepek AJ, Bachert C, Olze H. COVID-19 in a patient with severe chronic rhinosinusitis with nasal polyps during therapy with dupilumab. $J$ Allergy Clin Immunol. 2020;146 (1):218-220. doi:10.1016/j.jaci.2020.05.005

34. Ferrucci S, Romagnuolo M, Angileri L, Berti E, Tavecchio S. Safety of dupilumab in severe atopic dermatitis and infection of Covid-19: two case reports. J Eur Acad Dermatol Venereol. 2020;34(7):e303e304. doi:10.1111/jdv.16527

35. Caroppo F, Biolo G, Belloni Fortina A. SARS-CoV-2 asymptomatic infection in a patient under treatment with dupilumab. J Eur Acad Dermatol Venereol. 2020;34(8):e368. doi:10.1111/jdv.16619

36. Bhalla A, Mukherjee M, Radford K, et al. Dupilumab, severe asthma airway responses, and SARS-CoV-2 serology. Allergy. 2021;76 (3):957-958. doi:10.1111/all.14534

37. Rossi M, Rovati C, Arisi M, Soglia S, Calzavara-Pinton P. Management of adult patients with severe atopic dermatitis treated with dupilumab during COVID-19 pandemic: a single-center real-life experience. Dermatol Ther. 2020;33:e13765. doi:10.1111/dth.13765

38. Ordóñez-Rubiano MF, Campo I, Casas M. Dupilumab in atopic dermatitis, a protocol for SARS-COV-2-infected patients. Dermatol Ther. 2020;33:e14172. doi:10.1111/dth.14172
39. Ordóñez-Rubiano MF, Rubiano-Mojica PC, Casas M. Young HIV-positive male patient with severe atopic dermatitis on dupilumab and SARS-CoV-2 infection, a pioneer hypothesis. Int $J$ Dermatol. 2021;60(4):514-515. doi:10.1111/ijd.15499

40. Tanabe N, Matsumoto H, Hamada S, Ito I, Hirai T. Dupilumab maintenance therapy in an asthmatic patient with coronavirus disease 2019 pneumonia. Allergol Int. 2021;70(2):274-276. doi:10.1016/j. alit.2020.10.005

41. Chiricozzi A, Talamonti M, De Simone C, et al.; DA-COVID-19 study group. Management of patients with atopic dermatitis undergoing systemic therapy during COVID-19 pandemic in Italy: data from the DA-COVID-19 registry. Allergy. 2021;76(6):1813-1824. doi:10.1111/all.14767

42. Stingeni L, Hansel K, Antonelli E, et al. Atopic dermatitis in adolescents: effectiveness and safety of dupilumab in a 16 -week real-life experience during the COVID-19 pandemic in Italy. Dermatol Ther. 2021;34(5). doi:10.1111/dth.15035.

43. Hansel K, Patruno C, Antonelli E, et al. Dupilumab in adolescents with moderate to severe atopic dermatitis: a 32-week real-world experience during the COVID-19 pandemic. Clin Exp Dermatol. 2021. doi: $10.1111 /$ ced. 14862

44. Ceryn J, Niedźwiedź M, Skibińska M, Ciążyńska M, Lesiak A, Narbutt J. COVID-19 in patients with atopic dermatitis treated with Dupilumab: three cases and a literature review. Clin Cosmet Investig Dermatol. 2021;14:1131-1138. doi:10.2147/CCID.S321003

45. Menzella F, Ghidoni G, Galeone C, Capobelli S, Scelfo C, Facciolongo NC. Immunological aspects related to viral infections in severe asthma and the role of Omalizumab. Biomedicines. 2021;9 (4):348. doi:10.3390/biomedicines 9040348

46. Gonzales-van Horn SR, Farrar JD. Interferon at the crossroads of allergy and viral infections. J Leukoc Biol. 2015;98(2):185-194. doi:10.1189/jlb.3RU0315-099R

47. Gern JE. How rhinovirus infections cause exacerbations of asthma. Clin Exp Allergy. 2015;45(1):32-42. doi:10.1111/cea.12428

48. Teach SJ, Gill MA, Togias A, et al. Preseasonal treatment with either omalizumab or an inhaled corticosteroid boost to prevent fall asthma exacerbations. J Allergy Clin Immunol. 2015;136(6):1476-1485. doi:10.1016/j.jaci.2015.09.008

49. Sekine T, Perez-Potti A, Rivera-Ballesteros O, et al. Robust T cell immunity in convalescent individuals with asymptomatic or mild COVID-19. Cell. 2020;183(1):158-168. doi:10.1016/j.cell.2020.08.017

50. Lucas C, Wong P, Klein J, et al. Longitudinal analyses reveal immunological misfiring in severe COVID-19. Nature. 2020;584 (7821):463-469. doi:10.1038/s41586-020-2588-y

51. Donlan AN, Sutherland TE, Marie C, et al. IL-13 is a driver of COVID-19 severity. JCI Insight. 2021;6(15):e150107. doi:10.1172/ jci.insight. 150107

52. Poddighe D, Mathias CB, Brambilla I, Marseglia GL, Oettgen HC. Importance of basophils in eosinophilic asthma: the murine counterpart. J Biol Regul Homeost Agents. 2018;32(2):335-339.

53. Murdaca G, Di Gioacchino M, Greco M, et al. Basophils and mast cells in COVID-19 pathogenesis. Cells. 2021;10(10):2754. doi:10.3390/cells 10102754

54. Huang J, Zhang Z, Liu S, et al. Absolute eosinophil count predicts intensive care unit transfer among elderly COVID-19 patients from general isolation wards. Front Med. 2020;7:585222. doi:10.3389/ fmed.2020.585222

55. Farmani AR, Mahdavinezhad F, Moslemi R, et al. Anti-IgE monoclonal antibodies as potential treatment in COVID-19. Immunopharmacol Immunotoxicol. 2021;43(3):259-264. doi:10.1080/08923973.2021.1925906

56. Grieco T, Porzia A, Paolino G, et al. IFN- $\gamma /$ IL-6 and related cytokines in chronic spontaneous urticaria: evaluation of their pathogenetic role and changes during omalizumab therapy. Int J Dermatol. 2020;59 (5):590-594. doi:10.1111/ijd.14812 
57. Poddighe D, Vangelista L. Effects of omalizumab on basophils: potential biomarkers in asthma and chronic spontaneous urticaria. Cell Immunol. 2020;358:104215. doi:10.1016/j.cellimm.2020.10 4215

58. Ando T, Kitaura J. Tuning IgE: IgE-associating molecules and their effects on IgE-dependent mast cell reactions. Cells. 2021;10(7):1697. doi:10.3390/cells10071697

59. Pala D, Pistis M. Anti-IL5 drugs in COVID-19 patients: role of Eosinophils in SARS-CoV-2- induced immunopathology. Front Pharmacol. 2021;12:622554. doi:10.3389/fphar.2021.622554

60. Flores-Torres AS, Salinas-Carmona MC, Salinas E, RosasTaraco AG. Eosinophils and respiratory viruses. Viral Immunol. 2019;32(5):198-207. doi:10.1089/vim.2018.0150

61. Rodrigo-Muñoz JM, Gil-Martínez M, Sastre B, Del Pozo V. Emerging evidence for pleiotropism of Eosinophils. Int J Mol Sci. 2021;22:7075. doi:10.3390/ijms22137075
62. Damiani S, Fiorentino M, de Palma A, et al. Pathological postmortem findings in lungs infected with SARS-CoV-2. J Pathol. 2020;253:31-40. doi:10.1002/path.5549

63. Luecke E, Jeron A, Kroeger A, et al. Eosinophilic pulmonary vasculitis as a manifestation of the hyperinflammatory phase of COVID-19. J Allergy Clin Immunol. 2021;147(1):112-113. doi:10.1016/j.jaci.2020.09.026

64. Murao K, Saito A, Kuronuma K, Fujiya Y, Takahashi S, Chiba H. Acute eosinophilic pneumonia accompanied with COVID-19: a case report. Respirol Case Rep. 2020;8(9):e00683. doi:10.1002/rcr2.683

65. Smola A, Samadzadeh S, Müller L, et al. Omalizumab prevents anaphylactoid reactions to mRNA COVID-19 vaccine. J Eur Acad Dermatol Venereol. 2021;35(11):e743-e745. doi:10.1111/jdv.17549

66. Colaneri M, De Filippo M, Licari A, et al. COVID vaccination and asthma exacerbation: might there be a link? Int $J$ Infect Dis. 2021;112:243-246. doi:10.1016/j.ijid.2021.09.026
Journal of Inflammation Research

\section{Publish your work in this journal}

The Journal of Inflammation Research is an international, peerreviewed open-access journal that welcomes laboratory and clinical findings on the molecular basis, cell biology and pharmacology of inflammation including original research, reviews, symposium reports, hypothesis formation and commentaries on: acute/chronic inflammation; mediators of inflammation; cellular processes; molecular mechanisms; pharmacology and novel anti-inflammatory drugs; clinical conditions involving inflammation. The manuscript management system is completely online and includes a very quick and fair peerreview system. Visit http://www.dovepress.com/testimonials.php to read real quotes from published authors. 